\title{
LA REGLAMENTACIÓN DE LA PROSTITUCIÓN EN LA BARCELONA DE LA RESTAURACIÓN (1870-1890)
}

por

\author{
RAFAEL ALCAIDE GONZÁLEZ \\ Universidad de Barcelona
}

RESUMEN: El bigienismo fue una doctrina médica que incorporó al tratamiento de la enfermedad los aspectos sociales que estaban en relación directa con ella. Uno de los campos donde la doctrina bigiénica tuvo mayor incidencia fue en el ejercicio de la prostitución, pues a la práctica médica babitual se sumó tanto el estudio de las causas que determinaban su ejercicio como la inclusión de una serie de medidas profilácticas de contenido moral, mediante las que se pretendía controlar y moldear el comportamiento social y prevenir los efectos del contagio de las enfermedades venéreas y sifilíticas. Estos contenidos se plasmaron en las sucesivas reglamentaciones de la prostitución que se promulgaron en las ciudades más importantes de España, desde mediados del siglo XIX. En tales reglamentaciones se incluía la creación de las Secciones de higiene especial, destinadas a registrar, prevenir y controlar el ejercicio de la prostitución y las enfermedades que de él se derivaban. En Barcelona, durante la Restauración, la Sección de Higiene especial, presidida por Carlos Ronquillo, tuvo una especial incidencia en el tratamiento de la prostitución como enfermedad social y de la mujer prostituta como víctima de las precarias circunstancias sociales y laborales del proletariado, en la ciudad industrial de finales del siglo XIX.

Palabras clave: Prostitución. Reglamentarismo. Barcelona. Restauración. Carlos Ronquillo.

ABSTRACT: "Hygienism» was a medical doctrine which incorporated the social features that were directly related to disease into its treatment. One of the fields where bygienic doctrine bad most influence was on the exercise of prostitution. This was because the study of the causes that originated prostitution and the series of moral prophylactic measures that were introduced to balt prostitution were both added to medical practice. It was through these methods that the medical profession tried to control and shape social bebaviour and to prevent the effects of contagious venereal and syphilitic diseases. These elements were reflected in the regulation of prostitution that was adopted in the most important cities in Spain from the middle of the nineteenth century onwards. In this legislation the creation of Sections of special bygiene was included. These were designed to record, prevent and control the exercise of prostitution, as well as all the illnesses de-

Hispania, LXIV/3, núm. 218 (2004) 897-922 
rived from it. In the city of Barcelona, during the Restauration, the Section of special bygiene under Carlos Ronquillo's government focused particularly on the treatment of prostitution as a social disease and on the female prostitute as a victim of the precarious social and labour circumstances of the working class in the late nineteenth-century industrial city.

KEY WORDS: Prostitution. Regulation. The nineteenth century. Barcelona. Carlos Ronquillo.

La doctrina higiénica se desarrolló en Europa desde finales del siglo XVII. Este gran proyecto científico y social se fue gestando en los ambientes ilustrados de la época, especialmente en los círculos médicos del viejo continente, donde cada vez adquiría mayor importancia la preocupación causada por las frecuentes epidemias y enfermedades que asolaban Europa, causando tremendos estragos en la población. A estas calamidades periódicas se șumaron, desde mediados del siglo XVIII, los cambios propiciados por el desarrollo de la Revolución Industrial, y con ella del maquinismo y del sistema económico capitalista.

Estos avatares histórico-económicos determinaron la aparición de un nuevo conjunto de problemas sociales relacionados, por una parte, con la migración de grandes contingentes de población desde las áreas agrícolas hacia las ciudades, que iban incorporándose al nuevo proceso de industrialización y, por otra parte, un profundo cambio en las relaciones laborales, a partir del desarrollo de los nuevos sistemas de producción industrial, que redundaron en el hacinamiento, la explotación laboral y el deterioro físico de los contingentes humanos llegados a las ciudades y empleados como mano de obra no calificada en el proceso fabril. Dichos contingentes de población resultaban una presa fácil para las enfermedades relacionadas con la aparición de epidemias, pero también para aquellas enfermedades endémicas cuyo desarrollo estaba determinado por las condiciones de insalubridad de la ciudad, al igual que por el pauperismo y la desnutrición crónica en la que se veían obligados a vivir.

El higienismo no permaneció ajeno a esta problemática y, además de incorporar en su metodología las mejoras obtenidas a partir de los avances científicos que se iban produciendo en el tratamiento de las causas y efectos de las enfermedades, introdujo una innovación fundamental en la historia de la Medicina: la inclusión de los aspectos sociales causantes y derivados de la enfermedad como parte fundamental e inseparable de la misma. Esta vertiente social de la doctrina higiénica, que en un principio se basó en algunos de los aspectos más idealistas y utópicos del naciente ideario socialista, pronto derivó hacia posiciones más conservadoras, fuertemente impregnadas de un carácter burgués. Éstas determinaron que algunos de los aspectos técnicos más importantes de la doctrina, como eran la prevención o la profilaxis de la enfermedad, estuvieran en relación directa con unas reglas ético-morales de comportamiento social y personal, acordes con el optimismo racionalista y con el idealismo ro- 
mántico implícitos en la doctrina higiénica y en el reformismo burgués a que ésta se fue adaptando ${ }^{1}$.

De esta manera, el higienismo incorporó gran parte de los elementos contenidos en el discurso burgués tales como la conservación del capital social y el control y la racionalización del espacio urbano, y fue utilizado políticamente, de manera sesgada e interesada, como método para establecer una planificación y unas directrices que intentaban modelar una nueva sociedad en la que no tuvieran cabida, ni el peligro siempre latente de revueltas sociales, ni la presencia de elementos perturbadores, contrarios al sistema, como agitadores políticos, prostitutas, mendigos, criminales y vagos, entre otros. Desde esta perspectiva, no cabe la menor duda de que la doctrina higiénica fue en sumo grado intervencionista, pues sus efectos se hicieron patentes a través de múltiples aspectos relacionados con la legislación, la educación, el trabajo, la enseñanza, la vivienda, la manutención, la dieta, la higiene privada y pública, y la mayoría de los aspectos morales de la sociedad decimonónica.

El ámbito de aplicación más importante de la doctrina higiénica fue el espacio urbano. La ciudad reunía, en gran medida, todos aquellos aspectos que el higienismo trataba de mejorar, en especial aquellos relacionados con la proliferación de enfermedades debidas a la presencia de grandes concentraciones de población que se hacinaban en viviendas malsanas, situadas en barrios insalubres de callejuelas estrechas y malolientes, desprovistas de las más elementales medidas de higiene.

\section{EL INTERVENCIONISMO DE LA DOCTRINA HIGIÉNICA EN EL CONTROL DE LA PROSTITUCIÓN}

En España, las diversas crisis económicas y los numerosos cambios políticos, acaecidos a lo largo del siglo XIX, se hicieron especialmente patentes en las grandes ciudades españolas. Con la llegada de la Restauración borbónica en 1875 , la burguesía recuperó gran parte de las parcelas de poder perdidas durante el Sexenio revolucionario (1868-1874). El miedo a la aparición de nuevos conflictos sociales y a la insurrección popular se constituyó en la pesadilla cotidiana de la clase burguesa que no dudó, desde su primacía en los órganos de gestión o de gobierno, en aplicar un programa político de medidas estabilizadoras destinado a lograr una determinada paz social, que comportó un amplio recorte de las libertades individuales al que no era ajeno el ejercicio de la represión indiscriminada.

1 Ver ALCAIDE GONZÁLEZ; Rafael: «La introducción y el desarrollo del higienismo en Esspaña durante el siglo XIX. Precursores, continuadores y marco legal de un proyecto científico y social", Scripta Nova. Revista electrónica de Geografía y Ciencias Sociales (Barcelona), Vol. III, nº 50 (octubre de 1999). Dirección URL: (http://www.ub.es/geocrit/sn-50.htm).

Hispania, LXIV/3, núm. 218 (2004) 897-922 
Uno de los aspectos en que más se hizo patente el control del espacio urbano ejercido por la burguesía a través de los preceptos de la doctrina higiénica - de manera especial aquellos referidos a las actitudes morales-, fue el del ejercicio de la prostitución. Pero, a su vez, la óptica higienista determinó que las causas y efectos derivados de dicho ejercicio fueran interpretados también en clave social, al estudiarse no sólo las enfermedades derivadas de la prostitución, sino también las causas que determinaban que una mujer se convirtiera en prostituta, y que tenían su origen en las graves carencias de que adolecía la mujer de clase obrera, tales como la incultura, la falta de sustento y muy especialmente la explotación, la falta de trabajo y de emancipación, y la desigualdad de derechos respecto al hombre ${ }^{2}$.

El punto de contacto entre la prostitución y el higienismo, el campo de batalla donde se libró el arduo combate científico-moral contra la prostitución, fue la ciudad, pues en ella se desarrollaron las reglamentaciones de control sanitario y vigilancia policial de la prostitución y, como consecuencia de aquellas, la creación de Secciones de Higiene «especial», bajo la responsabilidad administrativa de los ayuntamientos o de los gobiernos de provincia.

En referencia a los aspectos clínico-patológicos derivados del ejercicio de la prostitución, cabe apuntar que el contagio de enfermedades de transmisión sexual (sífilis, blenorragias, etc.) tenía mayor incidencia en la franja de la población masculina comprendida entre los 20 y los 40 años, por lo que no es de extrañar que sus estragos preocuparan a los gobernantes al igual que a los patronos, que veían cómo una población joven, necesaria para el mantenimiento del ejército, la marina o la actividad productiva en su conjunto, iba menguando su número de efectivos en disposición de servir al conjunto de intereses públicos y privados de la Nación.

Por lo que respecta a los aspectos morales de la prostitución, pese a ser considerada como un «mal menor» desde el origen de las reglamentaciones en España, cabe reseñar que su ejercicio se consideraba una alteración del orden social burgués, una grave infracción de las normas de buen comportamiento que debían presidir las costumbres públicas, y un peligro para la institución familiar. Las prostitutas, al igual que los criminales, vagabundos, mendigos y vagos, representaban elementos marginales aunque peligrosos, que podían desestabilizar la racionalidad del sistema establecido; y, por ello, se convirtieron en las víctimas propiciatorias de un estricto e injusto control social, basado en la opresión y en la vigilancia de sus actividades.

2 Ver NASH, Mary: Mujer, familia y trabajo en España (1875-1936), Barcelona, Anthropos, 1983, pp. 7-60 (y en especial el apartado dedicado a la prostitución), y CAPEL MARTínEZ, Rosa María: «La prostitución en España: notas para un estudio socio-histórico», en Mujer y sociedad en España (1700-1975), Madrid, Ministerio de Cultura, 1982, pp. 269-298. Para la situación de la prostitución madrileña a finales del siglo XIX, es muy recomendable la consulta de la obra de DEL MORAl RuIZ, Carmen: La sociedad madrileña. Fin de siglo y Baroja, Madrid, Ed. Turner, 1974, al igual que el estudio de RIvière GómEZ, Aurora: Caídas, miserables, degeneradas. Estudio sobre la prostitución en el siglo XIX, Madrid, Comunidad de Madrid, Dirección General de la Mujer, 1994.

Hispania, LXIV/3, núm. 218 (2004) 897-922 
El intervencionismo de la doctrina higiénica, en referencia a los aspectos médicos y sociales del ejercicio de la prostitución, se puso de manifiesto a partir de tres corrientes de opinión que imperaron en mayor o menor medida en la actividad profesional de los médicos higienistas. Con todo, pese a sus diferencias, tales posicionamientos se veían, de algún modo, entremezclados en la práctica médica diaria, debido a la contradicción entre el pragmatismo científico y la importancia que los aspectos morales pudieran tener en la opinión personal del profesional. Estas corrientes de opinión fueron la prohibicionista, la reglamentarista y la abolicionista.

Bajo la postura prohibicionista, se argumentaba la ineficacia de la reglamentación y se proponían medidas de carácter represivo y punitivo, cuyo calado moral estaba en línea con los principios más ortodoxos de la religión católica. La prostituta era considerada mayoritariamente como reo de culpa.

La corriente de opinión reglamentarista consideraba por su parte la prostitución como «un mal menor», debido a la imposibilidad de impedir su práctica - tan habitual como extendida - mediante criterios prohibicionistas. Este carácter «inevitable» del comercio sexual determinó su tolerancia, pero a partir de criterios de racionalidad, es decir bajo determinadas normas que, basadas en criterios de policía sanitaria y moral, debían impedir la propagación de las enfermedades venéreas y sifilíticas derivadas de la prostitución. De esta manera, se hacía efectivo el control de las mujeres dedicadas a este oficio, al que se relacionaba con otras actividades delictivas, y se incorporaban, en parte, los criterios morales que propugnaban los partidarios de la prohibición. Sin embargo, la postura reglamentarista incorporaba notables diferencias, ya que se consideraba a la mujer prostituta como víctima, y por lo tanto, como susceptible de ser rehabilitada para incorporarse de nuevo a la sociedad.

Finalmente, con el criterio abolicionista, se pretendía demostrar que la moralización de ambos sexos por igual, a partir del restablecimiento de la moral cristiana y de las costumbres públicas, acabaría con el ejercicio de la prostitución, un ejercicio que no había disminuido, tanto por la ineficacia de la reglamentación (ya que ésta propiciaba el aumento de la prostitución clandestina) como por el carácter represivo y discriminatorio de la misma respecto a la mujer.

La postura que triunfó en España en buena parte de los siglos XIX y XX fue la reglamentarista. Ante lo arraigada que se hallaba la práctica de la prostitución en la sociedad, se admitió que la prohibición de su ejercicio y la clandestinidad a la que, con ella, se verían sometidas las mujeres prostitutas, causaría todavía más estragos en la expansión de las enfermedades venéreas; con lo cual, aceptada como un mal inevitable, se toleró y se reglamentó. En otro trabajo, hemos tratado del desarrollo de las reglamentaciones de la prostitución en España ${ }^{3}$, al igual que de los períodos en que su ejercicio estuvo prohibido.

3 ALCAIDE GONZÁLEZ, Rafael: «La higiene de la prostitución en Barcelona: una aproximación a los contenidos médico-sociales del higienismo en España durante el siglo XIX», Estudio introductorio a la reedición de la obra de Sereñana y PARTAGás, Prudencio: La prostitución en la ciudad de 
Como resumen, diremos que desde finales del siglo XVIII existen intentos de reglamentar el ejercicio de la prostitución (Cabarrús, 1792) y, ya en el siglo XIX, en la Exposición de Antonio Cibat (1809) ${ }^{4}$, y en el Proyecto de Reglamento general de Sanidad presentado por Mateo Seoane a las Cortes en 1822. Sin embargo, como considera acertadamente Jean-Louis Guereña, el arranque de la reglamentación de la prostitución en España puede fecharse a partir de 1854, durante el Bienio progresista, aunque algunos años antes, ya había tenido lugar la promulgación de dos documentos de gran importancia en el desarrollo del reglamentarismo en España: las Disposiciones de Zaragoza (1845), y el $R e-$ glamento para la represión de los excesos de la prostitución en Madrid (1847). Con posterioridad a estos documentos, se fueron promulgando una larga serie de reglamentaciones en gran parte de la ciudades españolas: Madrid (1854, 1859, 1863, 1865); Gerona (1854); Málaga (1855); Jerez de la Frontera (1855); Sevilla (1859); Alicante (1860); Cádiz (1861 y 1864); Santander (1862); Palma de Mallorca (1862); Barcelona (¿1863? y 1867); San Fernando (1864); Puerto de Santa María (1864); Valencia (1865) y Vigo (1867), entre otras 5.

Estas reglamentaciones estuvieron destinadas a «tolerar» de una manera racional aunque hipócrita el ejercicio de la prostitución. Su ámbito máximo de aplicación no superó los límites locales o provinciales. De sus contenidos legales cabe destacar tres grandes aspectos.

En primer lugar, los relacionados con las enfermedades y su tratamiento, ya que las líneas maestras de las diferentes reglamentaciones se basaron tanto en la lucha contra las enfermedades venéreas y en especial contra la sífilis, como en la necesidad de disminuir el elevado número de mujeres que ejercían la prostitución clandestina o privada. Sereñana y Partagás estimó al respecto, en 1882, que el número de mujeres que ejercían la prostitución clandestina en Barcelona era de 6.000, mientras que en Madrid la cifra ascendía a 17.000.

Barcelona estudiada como enfermedad social y considerada como origen de otras enfermedades dinámicas, orgánicas y morales de la población barcelonesa, Barcelona, Universidad de Barcelona, Geocrítica Textos electrónicos, $\mathrm{n}^{\circ} 2$ (noviembre de 2000). Dirección URL: (http://www.ub.es/geocrit/pspestin.htm).

4 Sobre Cabarrús y Cibat, ver los trabajos de GuEREÑA, Jean-Louis: "Los orígenes de la reglamentación de la prostitución en la España contemporánea. De la propuesta de Cabarrús (1792) al reglamento de Madrid (1847)», Dynamis, (Granada) no 15 (1995), pp. 401-441; y «Médicos y prostitución. Un proyecto de reglamentación de la prostitución en 1809: La «Exposición» de Antonio Cibat (1771-1811)", Medicina e Historia (Barcelona), $3^{a}$ época, $\mathrm{n}^{\circ} 71$ (1998), $16 \mathrm{p}$.

5 GUEREÑA, Jean-Louis: «Prostitución, Estado y Sociedad en España. La reglamentación de la prostitución bajo la monarquía de Isabel II (1854-1868)», Asclepio.(Madrid), Vol. 49 (1997), n 2, pp. 101-132. Un detallado estudio del desarrollo histórico de la reglamentación española en toda su extensión puede consultarse, entre otros, en los siguientes trabajos suyos: La prostitución en la España Contemporánea, Madrid, Marcial Pons, 2003, pp. 95-338; «La réglementation de la prostitution en Espagne aux $\mathrm{XIX}^{\mathrm{e}}$ et $\mathrm{XX}^{\mathrm{e}}$ siècles. Répression et réglementarisme», en CARRASCO, Raphaël (ed): La prostitution en Espagne de l'époque des Rois catholiques à la II République, Paris, Les Belles Lettres (Annales Littéraires de l'Université de Besançon, 526), pp. 229-257; «De bistoria prostitutionis. La prostitución en la España contemporánea», Ayer (Madrid), nº 25 (1997), pp. 35-72.

Hispania, LXIV/3, núm. 218 (2004) 897-922 
En segundo lugar, los aspectos de contenido moral y punitivo intentaban preservar el mantenimiento de las buenas costumbres y de la convivencia ciudadana, prohibiendo a las prostitutas cualquier manifestación pública de su condición, bajo pena de multa o cárcel, según fuese la gravedad de la infracción.

$\mathrm{Y}$, en tercer lugar, los aspectos de la relación administrativa de las meretrices y amas de mancebía con los poderes públicos fueron desarrollados de manera amplia en el articulado de dichos reglamentos, imponiéndose un buen número de multas y cuotas «sanitarias» de todo tipo a las prostitutas, como pago de la atención médica, hecho que constituía una apreciable fuente de ingresos adicionales para las arcas del Estado, representado por los Gobiernos Civiles y los Ayuntamientos.

Una de las consecuencias más importantes del desarrollo de estas reglamentaciones fue la creación de las llamadas Secciones de Higiene Especial, compuestas por personal médico, sanitario y administrativo, en las que se llevaron a cabo todas las actuaciones sobre el ejercicio de la prostitución que se regulaban en las reglamentaciones. Actuaciones que no estuvieron exentas de una acentuada polémica entre los cuerpos médico y administrativo, generada por el reiterado abuso burocrático en la aplicación de las cuotas y multas. Esta polémica motivó, en muchos casos, el desacuerdo de los médicos higienistas que quedó patente en el conjunto de reclamaciones y propuestas que éstos dirigieron a los diferentes alcaldes y gobernadores civiles, entre las cuales destacan las siguientes: la necesidad de la primacía de la atención médica frente a los aspectos administrativos, la supresión de la visita domiciliaria, la inscripción voluntaria y gratuita, la eliminación del impuesto y la creación de hospitales especiales. Aspectos contradictorios con el espíritu que emanaba de los reglamentos y que propiciaron, sin duda, que, tanto la atención médica como el trato humano dispensado a las prostitutas evolucionaran hacia posiciones más comprensivas de la difícil realidad social implícita en el mundo marginal de la prostitución.

\section{EL REGLAMENTARISMO EN BARCELONA. LA SECCIÓN DE HIGIENE ESPECIAL: APUNTES HISTÓRICO-SOCIALES}

Barcelona, desde mediados del siglo XIX, era una ciudad que experimentaba una eclosión industrial y urbana sin precedentes, acompañada de una floreciente actividad intelectual en todos los ámbitos del pensamiento. Por lo que respecta a la doctrina higiénica, el magisterio y la influencia ejercidos por personalidades como Pedro Felipe Monlau, Ildefonso Cerdà, o Pedro García Faria, entre muchos otros, determinaron que el proyecto intelectual higienista se plasmara en realidades concretas tales como el derribo de las murallas a partir de 1854 , el diseño de la nueva ciudad edificada a partir de aquellas, y la construcción de la red de alcantarillado, entre otras. Estas transformaciones urbanas 
estuvieron acompañadas de diversas actuaciones trascendentales en el campo cultural y científico como la creación de la Academia de Higiene de Cataluña en 1887, la celebración de la Exposición Universal de 1888, y la puesta en marcha del Inștituto de Higiene Urbana de Barcelona en 1891, dirigido por Luis Comenge.

Paralelamente a este desarrollo urbano, científico y cultural, la ciudad de Barcelona se convirtió en destino habitual de un considerable número de emigrantes, procedentes del mundo rural catalán y del resto de España que, acuciados por las malas cosechas o las hambrunas, intentaban encontrar en la capital catalana un mejor modo de subsistencia. Sin embargo, a pesar de que el ritmo de crecimiento industrial era cada vez más intenso, la pujante industria barcelonesa dependía también de las diversas coyunturas económicas y políticas que afectaban al conjunto de España, coyunturas cuyos altibajos tenían su reflejo social en las terribles fluctuaciones de un mercado de trabajo sin derechos reconocidos, que determinaban despidos masivos y, con ellos, el paro, el hambre, el hacinamiento y la miseria para gran parte de una población que, prácticamente, había duplicado los 190.000 habitantes con que contaba la ciudad Condal en 1860 .

Con el advenimiento de la Restauración, Barcelona vivió una época de renovación y estímulo de la actividad comercial: la llamada febre d'or. Sin embargo, este aumento de la prosperidad no activó la realización de las urgentes méjoras urbanas y sociales que necesitaba la ciudad. La escasez de medios de los recién llegados apenas les permitía instalarse más que en viviendas o habitaciones que no ofrecían un mínimo de salubridad, con dependencias estrechas y faltas de luz en las que tenían que malvivir, por lo general, a base de una dieta alimenticia absolutamente desequilibrada. Pero no acababan ahí las penurias de este sector de población obrera menos favorecido ya que, además, tenían que soportar las penosas e inseguras condiciones de trabajo que ofrecían las fábricas y los talleres, con jornadas que en algunos casos alcanzaban las 16 horas, el trabajo de menores y el despido libre, a lo que había que añadir el frecuente agiotaje de muchos de los productos de primera necesidad.

Todo este cúmulo de terribles y funestas consecuencias, propiciadas por los mecanismos utilitaristas del laissez-faire empleados por gobernantes y patronos, derivaban irremediablemente en el incremento de personas desocupadas que, al no contar con medios de subsistencia, no encontraban otra salida que la mendicidad o la prostitución. Con toda probabilidad, entre estos colectivos, el que más expectativas de trabajo y remuneración podía presentar, era el de las mujeres prostitutas, debido a la demanda sexual que generaba la presencia de un elevado numero de población masculina (trabajadora o desocupada) en la ciudad, al que además, se sumaba el contingente del personal que componía las guarniciones del ejército y de la marina militar y mercante ${ }^{6}$.

6 Ver ALCAIDE GONZÁleEZ, Rafael: «Inmigración y marginación. Prostitución y mendicidad en la ciudad de Barcelona de finales del siglo XIX: una comparación con la actualidad», Scripta

Hispania, LXIV/3, núm. 218 (2004) 897-922 
El establecimiento de una reglamentación sobre la prostitución y de su correspondiente Sección de Higiene especial tuvo lugar en Barcelona en 1863, cuando el gobernador civil Francisco Sepúlveda Ramos, que acababa de tomar posesión de la plaza, «deseando organizar la prostitución, al objeto de aminorar los estragos que la misma causaba en la salud pública, nombró una Comisión facultativa bajo la presidencia del distinguido práctico Dr. D. Juan Durán y Sagrera, a la sazón director de Sanidad marítima, encargándole la confección de un reglamento que, sosteniendo incólumes los principios de la moral, tendiera en lo posible a disminuir los estragos que la sífilis causaba en la población barcelonesa»?.

Algunos meses más tarde, en febrero de 1864 , le sustituyó el gobernador Antonio Guerola quien, con anterioridad, había ejercido las funciones de gobierno en las provincias de Sevilla, Cádiz y Málaga. Según relata el gobernador en su Memoria relativa a Barcelona, existía por aquel entonces un sistema reglamentarista que se basaba en el pago, por parte de las amas de mancebía y de las prostitutas, de los honorarios profesionales por los reconocimientos médicos que se les practicaban periódicamente. Según apunta Jean Louis Guereña, en 1864 - tras una reforma introducida por Guerola mediante la cual el dinero de los reconocimientos pasaba directamente a manos de los médicos, sin intermediarios - se contaban ocho médicos dedicados a estos menesteres que se repartían una recaudación de 8.000 reales semanales ${ }^{8}$.

En un artículo aparecido en La Independencia médica en 1878, el doctor Carlos Ronquillo y Morer (1838-1900) - uno de los mayores representantes del núcleo higienista barcelonés - exponía, en un artículo firmado bajo el pseudónimo de Teophilo Bombasto (bajo este seudónimo, el doctor Ronquillo sostuvo una larga polémica con el doctor Letamendi sobre la Gimnasia cristiana), que «en 1864 aparecieron entre nosotros las cartillas de las prostitutas»9. A renglón seguido, se preguntaba Ronquillo qué elementos eran los que inspiraban la reglamentación de la prostitución en Barcelona:

« ¿Será la doctrina o la moral católica? ¿Será acaso la libertad la inspiradora de nuestra reglamentación? ¿Qué memorias se han escrito? ¿Qué estadísticas se han publicado? ¿Qué piensa el público?».

De estas afirmaciones se desprende, en primer lugar, la probable existencia de una reglamentación, o tal vez de un documento de carácter interino que, al

\footnotetext{
Nova. Revista electrónica de Geografía y Ciencias Sociales (Barcelona), Vol V, $\mathrm{n}^{\circ} 94$ (agosto de 2001). Dirección URL: (http://www.ub.es/geocrit/sn-94-103.htm).

7 Sereñana y Partagás, Prudencio: La prostitución en la ciudad de Barcelona, op. cit., p. 99.

8 Guerola, Antonio: Memoria de mi administración en la provincia de Barcelona como gobernador de ella desde 5 de febrero hasta 14 de julio de 1864, cit. por GuerEÑA, Jean Louis: «Prostitución, Estado y Sociedad en España», op. cit., p. 123.

9 La Independencia médica (Barcelona), Año XIII (1878), no 11, pp. 129-130.
} 
igual que ocurrió con el resto de reglamentaciones sobre el ejercicio de la prostitución, estuvo inspirado en el que, en aquella época, estaba vigente en Madrid y, en segundo lugar, la reserva absoluta que presidía el tratamiento de los asuntos relacionados con la prostitución, y especialmente con el cobro de los diferentes arbitrios que se imponían a las prostitutas.

Desde luego, el nombramiento de la Comisión facultativa de 1863, y la afirmación del doctor Ronquillo antes citada, constituyen elementos suficientes para avalar la suposición de la existencia de una reglamentación en Barcelona desde 1863 o 1864 . Por otra parte, parece ilógico conjeturar que una ciudad de la importancia de Barcelona no dispusiera de una reglamentación sobre la prostitución en esa época. Sin embargo, lo cierto es que el primer documento que hemos podido localizar al respecto es el Reglamento para la vigilancia y servicio sanitario de las prostitutas de Barcelona promulgado por el Gobernador civil Romualdo Méndez de San Julián, fechado el 4 de noviembre de 1867, y cuyos articulado y contenidos son, en conjunto, muy similares a los ya comentados respecto a las reglamentaciones en general.

La convulsa sucesión de acontecimientos históricos en la política española determinó que en el período comprendido entre la fecha de promulgación del reglamento de Méndez de San Julián y diciembre de 1882, ocuparan el cargo de Jefe de la provincia de Barcelona veintiséis gobernadores civiles. Durante ese mismo período, se promulgaron dos nuevos reglamentos en 1870 y en 1874. Este último, con algunas modificaciones, era el que regía en 1882, y es el que reproduce Sereñana y Partagás en su trabajo sobre la prostitución en Barcelona que venimos citando.

Con los constantes relevos de gobernadores civiles, ni la higiene ni la administración pública podían desarrollarse con un mínimo de continuidad. Debido a esta caótica situación, la Sección de Higiene especial barcelonesa sufrió múltiples cambios en su sistema organizativo, al igual que en el personal facultativo y en el desarrollo de la actividad asistencial que se prestaba a las mujeres prostitutas, que dificultaron su normal funcionamiento. A todo ello, hay que añadir las frecuentes discrepancias, que ya hemos mencionado, del personal médico respecto al funcionamiento administrativo de las Secciones.

Todo este conjunto de adversidades determinó, a corto plazo, que muchas de las reivindicaciones médicas, tanto en materia de mejoras en la asistencia sanitaria, como en la necesidad de la eliminación de los impuestos y de la elección por méritos contraídos en el ejercicio de su profesión de los médicos higienistas destinados a la Sección, quedaran casi siempre postergadas para mejor ocasión. Veamos cómo se desarrolló una parte de la historia de la Sección de Higiene especial barcelonesa en relación con estas cuestiones.

Una de las necesidades más reclamadas para Barcelona, en materia de asistencia sanitaria, era la construcción de más centros hospitalarios, uno de los cuales estuviese destinado exclusivamente al tratamiento y curación de las llamadas enfermedades especiales o venéreas. El gran incremento demográfico 
que registraba Barcelona, de manera continuada, influyó en el aumento de enfermos que requerían hospitalización por dolencias de carácter general, en una ciudad que disponía de un solo hospital, el de la Santa Cruz, cuya capacidad se hallaba totalmente desbordada por la constante llegada de enfermos. Así relata Salvador Pinar, licenciado y antiguo alumno del Colegio de Medicina barcelonés, la situación de los enfermos sifilíticos:

«Barcelona, capital de 200.000 almas, esencialmente mercantil e industrial, cuenta en su seno con un solo hospital, exceptuando el de Lazarinos. Este hospital [el de la Santa Cruz], de pésimas condiciones, contiene dos departamentos de dementes, hospital general, hospital clínico y hospitales especiales de enfermedades sifilíticas y de los órganos de la visión. [...] No existiendo más que un solo hospital para toda clase de enfermos, ha de quedar una pequeñísima parte para los que padecen enfermedades sifilíticas. [...] Entre los departamentos de ambos sexos escasamente se cuentan 100 camas y aún éstas colocadas de tal modo y en tales salas, que no se puede penetrar impunemente en ellas, si antes no se ha tomado la precaución de airearlas convenientemente [...]. Además, como 100 camas no son número suficiente para contener todos los enfermos sifilíticos que se presentan para entrar en el hospital, tiene que establecerse un turno de espera, marchándose los enfermos a implorar la caridad pública cuando no disponen de alguna boardilla» 10 .

En 1880, a efectos de control de la prostitución, la ciudad de Barcelona se hallaba dividida — según describe Sereñana y Partagás_-, en nueve distritos, y en el dispensario de Sanidad, sito en. la Rambla de Santa Madrona número 30, se atendían todos los martes y jueves por la tarde; las mujeres que no tenían cabida en el hospital de la Santa Cruz ${ }^{11}$, ya que en esta institución se habilitaban, únicamente, 55 camas para mujeres enfermas de venéreo y 53 camas para hombres enfermos de la misma afección. La insuficiente estructura de los recursos asistenciales propiciaba que gran parte de las mujeres prostitutas que carecían de recursos y que, por el escaso número de camas disponibles, no podían ingresar en las salas de venéreas del hospital de la Santa Cruz, no tuvieran otro remedio que curarse en sus propios domicilios. Como apunta Sereñana, «estas mujeres, para poder atender al pago de la manutención, vestir, cuota sanitaria y demás, se ven en la imperiosa necesidad de admitir a cuantos individuos se les presenten ${ }^{12}$.

Esta terrible situación personal repercutía peligrosamente en la propagación de las enfermedades venéreas y hacía inútiles todos los esfuerzos previos en el control de la enfermedad de la prostituta ya que, si no estaba completamente curada, podía seguir transmitiendo la enfermedad a cualquier individuo con

10 PinAR, Ldo. Salvador: «Los sifilíticos en Barcelona», La España Médica (Madrid), 1863, pp. 61-62.

11 Sereñana y Partagás, Prudencio: La prostitución en la ciudad de Barcelona, op. cit., pp. 102-103.

12 Ibid., p. 151. 
el que mantuviese relaciones. En el caso de que la prostituta tuviera suficiente dinero, le era permitido curarse en su domicilio, siempre bajo la supervisión, bien de los médicos de la Sección de Higiene especial, bien de cualquier otro médico de su confianza. En este último caso, debía presentar certificado semanal firmado por el médico en cuestión. No ocurría así con las mujeres prostitutas sin recursos económicos.

Otro aspecto, relacionado con el ejercicio de la profesión médica, que dificultó la relación entre los médicos y los poderes civiles, fue el sistema de provisión de plazas de médicos higienistas destinados a la Sección de Higiene especial barcelonesa. Las denuncias manifestadas por Sereñana o Bassols y Prim, entre otros autores, de las frecuentes infracciones al respecto, indican que la asignación de estas plazas no siempre estuvo en correspondencia a los méritos de los profesionales que las detentaron. Las plazas de médicos higienistas, dado el especial carácter de su desempeño, estuvieron casi siempre en manos del poder político y fueron utilizadas en muchas ocasiones como prebendas y pagos de favores debidos. Así las cosas, una determinada situación política podía favorecer la adquisición de determinadas plazas de médico higienista por parte de individuos sin la debida experiencia profesional, situación que incidía negativamente en la siempre difícil continuidad en el quehacer médico, sobre un tema tan delicado como la prostitución y sus consecuencias, y que anteponía la influencia que las autoridades políticas pudieran ejercer desde el Gobierno civil o el Ayuntamiento sobre el desarrollo científico y social de la doctrina higiénica en toda su amplitud.

Así, en 1884, ante las continuas suspicacias a que se prestaba la asignación de plazas de médicos higienistas, el gobernador civil Aquilino Herce decidió acabar de una vez por todas con aquella situación, convencido de que «para dar respetabilidad y estabilidad al nombramiento de los médicos que debían desempeñar cargos de tal naturaleza, debía, no sólo asesorarse, sino confiar el asunto a personas de valor y de representación» ${ }^{13}$. Para ello, convocó una Comisión asesora, integrada por los doctores Bartolomé Robert (Presidente de la Real Academia de Medicina y Cirugía), Juan de Rull (Decano de la Facultad de Medicina) y Rafael Rodríguez Méndez (Catedrático de Higiene de dicha facultad), y presidida por Leonardo Casaña, Rector de la Universidad Literaria barcelonesa.

Esta Comisión redactó un informe en el que se proponía, en primer lugar, la provisión de las vacantes existentes, mediante «riguroso orden de antigüedad y que para las resultas no haya otra puerta de entrada que la oposición ${ }^{14}, y$, en segundo lugar, la candidatura con los nombres y cargos de los médicos integrantes de la Sección de Higiene especial barcelonesa, que fueron los siguientes: Presidente: Carlos Ronquillo y Morer; Vicepresidente: Federico Castells y

13 Bassols y Prim, Agustín: «La Sección de Higiene especial en Barcelona», Revista de Higiene y Policía Sanitaria (Barcelona), Vol. I (1890), p. 30.

14 Ibid, p. 31.

Hispania, LXIV/3, núm. 218 (2004) 897-922 
Ballespí; Médicos: Luis Suñé y Molist, Ángel Moya Caragol, Agustín Bassols y Prim, Pedro Verdós y Mauri, Isidro Calvet y Nava; Suplentes: José Corominas y Sabater, Juan Durich y Ferrando, Luis Cortés y Aguiló y Pablo Torras y Pascual. Todos ellos reunían, según la Comisión «sobrados conocimientos para el cargo, y su acrisolada moral y no desmentida honradez, son una garantía [...]. Todos tienen la independencia de carácter necesaria para tan delicado cargo, y muchos de ellos gozan de una posición social bastante desahogada para hallarse al abrigo de toda sospecha» 15 .

De esta forma, se zanjó temporalmente la cuestión de la provisión de plazas en la Sección. Esta situación duró hasta 1889, en que por Real Orden de 4 de enero del mismo año se traspasaban todos los servicios relacionados con la higiene a los Ayuntamientos. Con motivo de este traspaso de funciones, el alcalde Rius y Taulet encargó de inmediato a Juan Giné y Partagás — una de las personalidades médicas más influyentes de la época, a través de sus facetas política, periodística y publicista ${ }^{16}$ - , proponer la organización definitiva del servicio de Higiene y el replanteamiento interino del ramo de Sanidad municipal.

El resultado de este encargo quedó reflejado en el oficio de 2 de febrero de 1889 , enviado por Rius y Taulet a Carlos Ronquillo, en el que se puede leer lo siguiente:

\begin{abstract}
«Organizado por esta Alcaldía en virtud de autorización que al efecto me ha sido concedida el servicio de Higiene especial, de que a consecuencia de las recientes disposiciones dictadas por el Gobierno se ha hecho cargo el Excmo. Ayuntamiento de mi presidencia, en el día de hoy tomarán posesión de sus destinos los facultativos para aquel objeto designados por mi autoridad y habrán, por consiguiente, de cesar en el desempeño de dicho cometido los que constituyen el Cuerpo médico higienista que $\mathrm{V}$. dignamente ha presidido y fueron nombrados por el Gobierno de la Provincia».
\end{abstract}

De esta manera, tan absurda como repentina, se cesó a todos los componentes del Cuerpo médico de la Sección de Higiene especial que habían sido designados por la Comisión médica independiente en 1884, interrumpiendo la continuidad en la labor de atención médica que se venía llevando a cabo. Desafortunadamente, se puede comprobar de nuevo cómo aparecen el favoritismo político y la designación "por mi autoridad» que otrora emplearan los gobernadores civiles a la hora de asignar las plazas de higienistas.

Además, en relación con lo anterior, cabe anotar otro aspecto de carácter corporativo implícito en el funcionamiento de las Secciones de higiene especial, ya que el cuerpo médico era el único que, profesionalmente, se sentía cualificado para reformar los reglamentos pues se consideraba, mayoritariamente, que

15 Ibid.

16 Ver ALCAIDE GoNŹ́LEZ, Rafael: «La introducción y el desarrollo del higienismo en España durante el siglo XIX», op. cit. 
los aspectos burocráticos y administrativos, ciertamente desacreditados, o bien debían relegarse a un segundo plano o bien desaparecer de la reglamentación. Sin embargo, estas reivindicaciones topaban con la potestad que detentaba el gobernador civil de modificar los reglamentos en los puntos que considerase oportuno. Esta potestad, que emanaba de su representación como autoridad máxima en materia civil, nos hace suponer que, ante los diversos altibajos políticos, las redacciones de los reglamentos estuvieron impregnadas del espíritu político del momento en que se llevaban a efecto, especialmente en un tema —el de la prostitución en su relación con la moral y las costumbres públicas- que se convertía en un recurso político de gran magnitud.

De esta manera, la Administración permaneció insensible a las reiteradas demandas de los médicos higienistas en cuanto a la reforma de algunos de los artículos más discutidos de las reglamentaciones, como la inscripción o las bajas de las prostitutas en los Registros y el pago de arbitrios, tasas y multas por diversos conceptos, debido a la considerable fuente de ingresos que dichos tributos representaban para las arcas de.los gobiernos civiles, a tenor del número de mujeres inscritas en las Secciones de higiene especial:

«En el Gobierno Civil se vio claro que la inspección y reglamentación del servicio de higiene llamada especial, podía ser la fuente de ingresos no despreciables y que podía coadyuvar a sufragar los gastos que aquel importaba, además de los honorarios a los facultativos que desempeñasen los cargos de higienistas».17.

El doctor Carlos Ronquillo denunciaba con rotundidad aquella injusta situación:

«Fijar impuestos a la infamia, a la miseria, a la degradación, a la inmundicia y a la pobreza, es un acto atentatorio a toda noción de justicia, a todo sentimiento bello y bueno. Por idénticos motivos que la meretriz deberían pagar contribución el pobre para pedir limosna, el ladrón para dedicarse a sus atentados, el apestado para comunicar la muerte y hasta el moribundo para morirse [...], la reglamentación debería formar parte de una vasta institución social dedicada a impedir el desarrollo de la prostitución, a velar continuamente por la mejora de las prostitutas sorprendiendo un momento y una ocasión oportuna para redimirla, sin que un ama pudiera alegar deudas de ningún género. En una Reglamentación perfecta que mereciese el aprecio del público y el respeto de las meretrices, no deberían mencionarse ni derechos de inscripción, ni cuotas mensuales, ni tan siquiera multas»18.

Como se ha visto líneas atrás, no faltaron aspectos conflictivos en el quehacer profesional de los higienistas adscritos a la Sección de Higiene especial bar-

17 BASSOLS Y Prum, Agustín: «La Sección de Higiene especial en Barcelona», op. cit., p. 29.

18 RONQUillo MORER, Carlos: «En una reglamentación perfecta no debe admitirse el oro de las prostitutas. Discurso leído ante el Cuerpo de Médicos higienistas el 23 de febrero de 1885", Gaceta Médica Catalana (Barcelona), Año IX (1886), nº 206, pp. 35-40. 
celonesa; aspectos que, por otra parte, estuvieron con toda seguridad presentes en las Secciones de la mayoría de ciudades españolas. Resultaba ciertamente difícil conjugar una atención digna y profesional en el tratamiento de las enfermedades derivadas del ejercicio de la prostitución, con la falta de medios físicos y humanos para desarrollarla en su integridad. En muchos casos, sólo la motivación profesional y la tenacidad inagotable de muchos médicos higienistas para aportar soluciones a los problemas médicos y sociales de las prostitutas, superaron el tremendo escollo impuesto por la indiferencia administrativa y por el oportunismo político implícito en el funcionamiento de estas Secciones.

Así ocurrió en la Sección de Higiene especial de Barcelona, presidida por el doctor Carlos Ronquillo y Morer entre 1881 y 1889. Durante esos fructíferos años, la labor desarrollada por los médicos higienistas adscritos a la Sección tuvo importantes manifestaciones, tanto en el desarrollo de la estadística asistencial, como en el tratamiento médico y social de la prostitución, a partir de la publicación de innumerables artículos sobre tan arduo tema y de la creación, en 1888, del Dispensario para enfermedades especiales de Nuestra Señora de las Mercedes. A estas realizaciones contribuyó, sin duda, la toma de posesión del gobernador civil Luis Antúnez y Monzón en 1884, quien, además de mantener unas relaciones personales inmejorables con Carlos Ronquillo, permitió que, a lo largo de su mandato, primasen los criterios médicos frente a los puramente administrativos, en la organización y el funcionamiento de la Sección de Higiene especial barcelonesa. Veamos de qué manera influyó este notable compromiso personal, y en qué aspectos se concretó tan ansiada como reivindicada mejora en la atención médica de las enfermedades venéreas y sifilíticas.

\section{EL DOCTOR CARLOS RONQUILLO Y LA CREACIÓN DEL DISPENSARIO PARA EN- FERMEDADES ESPECIALES DE NUESTRA SEÑORA DE LAS MERCEDES}

La atención médica y el trato personal que se prestaban a las mujeres prostitutas en las Secciones de higiene especial, dependía en gran medida del profesional que las dispensaba. No parece descabellado apuntar que el contacto diario con la terrible realidad de la prostitución, con las grandes diferencias sociales que existían entre las prostitutas más adineradas o las pobres de solemnidad, con el conocimiento de las causas por las que muchas de aquellas mujeres ingresaban en el mundo de la prostitución y con las continuas corruptelas que encubría el cobro de los servicios prestados, influyera de alguna manera en los médicos higienistas, determinando un mayor o menor interés de éstos en el desempeño de su difícil trabajo diario.

Una empresa, un proyecto, dependen muchas veces de la sensibilidad, del carácter de la persona que los lidera. En el caso de las Secciones de higiene especial donde, como hemos visto, no siempre primaron los conocimientos médicos sobre el oportunismo político, y por ello se concedieron plazas de médicos 
numerarios y supernumerarios sin otros requisitos que la afinidad política o el favor debido, parece claro que la vocación profesional y la ética personal de cada uno de los componentes del cuerpo médico higienista tenían que determinar el mejor o peor funcionamiento de estos centros de asistencia médica. Así las cosas pese a estar regidas por una reglamentación similar, cada Sección de Higiene especial pudo tener un desarrollo histórico distinto, marcado por la impronta personal de sus directores o presidentes, en la medida en que éstos disfrutaron, por parte de los gobernadores civiles o los alcaldes, de la comprensión, de la libertad y de la independencia en su labor médica y en el tratamiento del terrible problema de la prostitución y sus consecuencias.

Este fue el caso del doctor Carlos Ronquillo y Morer, presidente de la Sección de Higiene especial barcelonesa desde 1881 hasta 1889. Hijo del ilustre químico y farmacéutico José Oriol Ronquillo y Vidal —exilado liberal, primer Presidente del Colegio de Farmacéuticos de Barcelona y autor del famoso Diccionario de materia médica, industrial y agrícola-, Carlos Ronquillo y Morer se licenció en Medicina en 1858, doctorándose en Barcelona en 1869, e iniciando su labor médica en el ejercicio de la pediatría. Sus inquietudes intelectuales, sus ideales democráticos y sus excelentes relaciones con Ildefonso Cerdà y Pedro Felipe Monlau, le llevaron a formar parte entre 1865 y 1875, como vocal médico, de la Junta de Ensanche de Barcelona, desde donde desarrolló una amplia labor crítica a propósito de las continuas mutilaciones urbanísticas que sufrió el primitivo Proyecto de Ensanche de Cerdà, por razón de la tremenda especulación que los agentes económicos ejercieron sobre el espacio donde se debía construir la nueva Barcelona, «combatiendo con entusiasmo las mezquinas teorías de los defensores del miserable ochavo, del insano egoísmo y de la codicia sin entrañas» ${ }^{19}$.

En el mismo año en que entró a formar parte de la Junta del Ensanche de Barcelona, presentó su candidatura a socio numerario de la Academia de Medicina y Cirugía de Barcelona, mediante la lectura de una Memoria titulada Consideraciones sobre la necesidad de la intervención de la Medicina legal en los tribunales excepcionales y acerca de la importancia de conceder más facultades al médico en todos los tribunales para la investigación de la verdad. Su candidatura fue rechazada por lo avanzado de las ideas políticas que en ella exponía, lo que representó para Ronquillo uno de los primeros reveses institucionales en su vida profesional.

Sin embargo, esta injusta situación no impidió que el higienista barcelonés formara parte de diversas sociedades médico-científicas, como la Médico Quirúrgica Matritense, las de Medicina y Cirugía de Castilla la Vieja y de Valencia, el Instituto de Vacunación y la Real Academia de Ciencias médicas de Palermo, la Médico-Quirúrgica de Génova, la Sociedad de Agricultura, Ciencias y Artes de Poligny, la Sociedad frenopática italiana y la Sociedad Imperial de Medicina de Constantinopla; siendo, además, miembro de la Sociedad Económica Barcelonesa

19 HeRP, J.: «El Dr. D. Carlos Ronquillo y Morer. Apuntes necrológicos», Gaceta Sanitaria de Barcelona (Barcelona), 1900, p. 68.

Hispania, LXIV/3, núm. 218 (2004) 897-922 
de Amigos del País, y compaginando, a lo largo de su vida, estos cargos con los de médico forense de la Curia eclesiástica, facultativo del Cuerpo de bomberos de Barcelona y miembro de la Academia de Higiene de Cataluña.

Pero no termina aquí la encomiable labor médica y humana llevada a cabo por Carlos Ronquillo, ya que durante el período revolucionario expuso en público el tema titulado Educación bigiénica de un pueblo, una de sus obras más trascendentales. En 1870, la Diputación provincial de Barcelona le nombró Inspector higienista de la provincia. En ese mismo año, el Ayuntamiento le concedió la Medalla de plata por los servicios desempeñados durante la epidemia de fiebre amarilla y su obra titulada Preceptos de salubrificación y beneficencia aplicables a las epidemias de Barcelona en general y a las de fiebre amarilla en particular, y fue nombrado director del hospital de coléricos de la Vinyeta. Un año más tarde, fue designado miembro del jurado calificador de la Sección alimenticia en la Exposición de 1871 , y formó parte de la Junta nombrada para dictaminar sobre los planos del Parque y jardines que se tenían que construir en los antiguos terrenos ocupados por la Ciudadela, participando además, junto con el doctor José de Letamendi en el dictamen sobre la Instalación de un Matadero en Barcelona.

Dos años más tarde, en 1873, con el advenimiento de la República, el doctor Carlos Ronquillo fue elegido concejal del Ayuntamiento barcelonés mediante sufragio popular, responsabilidad política que asumió con la determinación que siempre le caracterizó y que supuso diversas mejoras en la salubridad e higiene ciudadanas y en la conservación del patrimonio artístico de la ciudad Condal. Algunos años más tarde, entre 1883 y 1887 , ejerció de vocal de la Junta local de Primera Enseñanza, siendo nombrado vicepresidente de Higiene del Congreso Médico de 1888. Y, al año siguiente, logró la Medalla de oro en la Exposición de Higiene de la Infancia celebrada en París.

A su fecunda actividad profesional, Carlos Ronquillo aunó su faceta de escritor y periodista, fundando y dirigiendo - por iniciativa de Pedro Felipe Monlau - la revista El Monitor de la Salud (que intentó ser una continuación de la que Monlau había editado con el mismo nombre años atrás) y publicando numerosos artículos relacionados con la higiene y la pediatría en periódicos y revistas científicas como La Independencia médica, la Revista de Higiene y policía Sanitaria, El Pabellón médico, la Crónica de Higiene y Terapéutica, la Crónica de enfermedades de los niños, el Boletín de la Academia de Higiene, El Monitor de la primera enseñanza, la Gaceta Médica Catalana, la Crónica de enfermedades de los niños, el mencionado Monitor de la Salud y La Gaceta Sanitaria de Barcelona, entre otras, artículos en los que utilizó en algunas ocasiones los pseudónimos de Teresa Coronado y Teophilo Bombasto.

En cuanto a su obra escrita, cabe destacar, entre otros, los siguientes títulos: Los obreros de Barcelona. Consideraciones generales (1862); El Consultor bigiénico y médico de las madres de Familia (1865); Consideraciones sobre la necesidad de la intervención de la Medicina legal en los tribunales excepcionales y acerca de la importancia de conceder más facultades al médico en todos los tribunales para la investigación de la verdad 
(1866); División de los establecimientos industriales (1869); Notas necrológicas del doctor Pedro Felipe Monlau (1871); El Manicomio del presente y del porvenir; Educación bigiénica de un pueblo (1870); Preceptos de salubrificación y beneficencia aplicables a las epidemias de Barcelona en general y a las de fiebre amarilla en particular (1871); Cédula infantil (1872); Adiciones a la gimnástica bigiénica de Lladó (1876); Armonías de la bigiene con el catecismo de la Fe católica (1876); Primeras necesidades del bombre. Los alimentos (1877); Sintomatología infantil. Notas compiladas (1880); Diferenciación de los procesos morbosos encefálicos de los niños (1889).

Hemos dejado para el final, deliberadamente, la fecunda labor médica y humana llevada a cabo por el doctor Carlos Ronquillo y Morer con respecto a la prostitución barcelonesa. Desde 1881 hasta 1889, época en la que ostentó el cargo de presidente de la Sección de Higiene especial de Barcelona, dicha Sección dependía del Gobierno civil de la provincia. Con la promulgación de la Real Orden de 4 de enero de 1889, todos los asuntos relacionados con el control de la prostitución fueron traspasados a los Ayuntamientos, con lo que - como ya hemos visto con anterioridad- se produjeron cambios en el personal médico de la Sección que afectaron notablemente a su desarrollo, inspirados por la influencia de Juan Giné y Partagás en el Consistorio barcelonés.

Durante los ochos años de permanencia al frente de los destinos de la Sección de Higiene especial de Barcelona, Carlos Ronquillo no se limitó únicamente al estricto cumplimiento de sus funciones como responsable de la Sección, como cabría esperar de la versatilidad y amplitud de sus múltiples cargos y de su bien ganada fama. Al contrario, el higienista barcelonés se dedicó por completo, con la inestimable ayuda de sus colaboradores, a la mejora en la atención sanitaria que se prestaba a las prostitutas, y, lejos del estigma reglamentarista y de las consideraciones morales al uso, hizo gala de un pragmatismo científico y de un discurso sociológico únicos en la trayectoria del tratamiento de la prostitución y sus consecuencias en la ciudad Condal.

De la lectura de sus innumerables artículos, entre los que cabe destacar los titulados «En una reglamentación perfecta no cabe el oro de las prostitutas», «Inscripción de las prostitutas», "Interrogatorio de las prostitutas», y las sucesivas Memorias sobre el estado de la prostitución llevadas a cabo por la Sección de Higiene especial desde 1884, entre otros, se desprende, en primer lugar, la profunda aversión de Ronquillo hacia la explotación humana que sufrían las mujeres prostitutas por parte de las amas de mancebía que «a semejanza de jefes que fueron reclutas, acostumbran a ser crueles con sus antiguas compañeras de infortunio» y «se distinguen por su astucia para ocultar las enfermas y por su crueldad en obligarlas a que propaguen el contagio» ${ }^{20}$.

Apuntemos, en segundo lugar, la denuncia constante de la inhibición política y social frente a tan terribles situaciones:

20 RONQUILlo MORER, Carlos: «Interrogatorio de las prostitutas», Gaceta Sanitaria de Barcelona (Barcelona), 1892, pp. 226-227.

Hispania, LXIV/3, núm. 218 (2004) 897-922 
«No, la prostitución no es una industria. La prostitución es una plaga social; la prostituta es una pobre de espíritu y de bolsa, expuesta siempre a contraer enfermedades especiales para comunicarlas a su vez. Los gobiernos, si quieren reglamentar la prostitución, no deben limitar sus aspiraciones a limitar la propagación de los males venéreo y sifilítico sino que deben velar por las jóvenes expuestas a la seducción, tender una mano amiga a la seducida y hasta procurar que retroceda la más degradada» 21 .

Y, en tercer lugar, se puede observar la comprensión y la conmiseración sinceras hacia las causas que motivaban el ingreso en la prostitución de muchas mujeres que carecían de otros medios con los que ganarse el sustento; todo ello cuestionado a partir de una firme independencia de criterios, que siempre acompañó la actuación profesional de Carlos Ronquillo al frente de la Sección de Higiene especial barcelonesa:

«¿Cómo exigir pesetas y más pesetas a la pobre que, para ganar una fracción de peseta, recorre calles, se desliza por escalerillas, atraviesa plazas y se detiene en derruido foso o bajo la barca del pescador? Está obligada a pagar una contribución y no tiene dinero. ¿Cómo saldrá de tal apuro? [...] En donde la reglamentación impera, no es el ama, si resulta cruel y ladrona, la causante de las peripecias, siempre con pérdidas, de la vida de la prostituta, sino la Administración por exigir derechos a una pobre, a una mísera, a una mendiga, y por no saber impedir la explotación»22.

No cabe la menor duda de que el trato diario y el conocimiento de las múltiples causas que determinaban la tremenda situación social de muchas de las mujeres prostitutas que se atendían en la Sección - a las que él mismo interrogaba en su calidad de presidente-, al igual que sus profundas convicciones cristianas, influyeron decisivamente en la forma como trató el problema de la prostitución.

A diferencia del reglamentarismo estricto de su coetáneo y colega Prudencio Sereñana y Partagás o del publicismo, muchas veces oportunista, de Juan Giné y Partagás, los escritos de Ronquillo revelan una preocupación por la mejora de las condiciones de vida, no tan sólo de la prostituta, sino de la mujer proletaria en su conjunto; y si bien es verdad, que en ocasiones están impregnados de cierto tinte moralizante, no es menos cierto que también incorporan un componente sociológico fuera de lo común, en el que destacan múltiples alegatos respecto a la emancipación de la mujer como manera de abolir la pros-

21 RONQUillo MORER, Carlos: «En una Reglamentación perfecta no debe admitirse el oro de las prostitutas", op. cit., Pp. 38-39.

22 RONQUillo MORER, Carlos: «Inscripción de las prostitutas. Discurso leído en la Academia de Ciencias Médicas el 5 de noviembre de 1885», Gaceta Médica Catalana (Barcelona), Año IX (1886), p. 674. 
titución, que incorporan una tremenda carga de humanidad basada en una absoluta piedad y respeto:

«Para las infelices que con la prostitución ganan su pan para ellas y tal vez para un ser querido [....] respeto, compasión. No inscripción rápida que a castigo se asemeje: al contrario, apurar todos los recursos de la caridad cristiana o de la más razonada filantropía si el primer concepto disgusta a ciertos paladares. ¿Qué investigaciones se practican sobre los antecedentes, vida y costumbres de la reo de inscripción? ¿Qué correcciones se imponen? ¿Qué consejos se dan? ¿Qué recursos se proporcionan? La nulidad es la respuesta» ${ }^{23}$.

La actividad de la Sección de higiene registró significativos cambios en su funcionamiento durante los años de presidencia de Carlos Ronquillo. Entre ellos cabe destacar los siguientes: la elaboración regular de estadísticas médicas; el estudio y la reforma de la reglamentación sobre la prostitución; la realización de oposiciones al cuerpo médico higienista y la formación del tribunal examinador en el que figuraron al menos tres de los componentes del Cuerpo médico de la Sección; la prestación de servicios relacionados con la epidemia de cólera en 1885, y servicios de carácter general como «informes sobre las casas de dormir, sobre la cárcel, sobre contagios, etc. Tanto en Barcelona como en Tarrasa, Manresa y otros puntos de la provincia» 24; la fundación de una Junta Benéfica destinada al sostenimiento y recaudación de los fondos necesarios para la creación del hospital de enfermedades especiales; la creación de un servicio de atención médica a las nodrizas, ante la posibilidad de transmisión de enfermedades contagiosas (la nodriza debía estar exenta de enfermedades, tanto de las que podían contagiarse inmediatamente, como de las que por medio de la leche podían imprimir al organismo del niño un sello nativo de debilidad) ${ }^{25}$.

$\mathrm{Y}$ así un largo etcétera que concluye con la creación del pequeño dispensario de Nuestra Señora de las Mercedes, sito en la calle Berenguer el Viejo número 6 de la ciudad Condal y destinado a la curación médica y a la rehabilitación social de las prostitutas enfermas. El dispensario fue inaugurado el domingo 16 de septiembre de 1888, con la asistencia de un gran número de médicos participantes en el Congreso Médico que se celebraba entonces en Barcelona, por el gobernador civil Luis Antúnez. La Vanguardia ofrecía, al día siguiente, esta reseña:

«Sacando todo el partido posible de las condiciones del local, queda instalada una enfermería con veinticinco camas construidas ad boc, puestas en amplio salón

\footnotetext{
23 Ibid., p. 670.

24 BASSOLS Y Prim, Agustín: «La Sección de Higiene especial en Barcelona», op. cit., p. 64.

25 Ver «Circular y Reglamento aprobados en 10 de diciembre de 1888 por el Excmo. Sr. Gobernador Civil de esta provincia. D. Luis Antúnez y Monzón», Revista de Higiene y Policía Sanitaria (Barcelona), vol. I (1890), p. 10.
} 
ventilado por tres grandes balcones y cuyo pavimento cubre muelle alfombra [...], cuarto de baño, cuarto de reconocimientos, comedor, sala de labores (con máquina de coser, costureros y veladores); habitación para enfermeras y vigilantes; oficinas dotadas de todos los recursos encaminados al mejor servicio; todo esto ha logrado ver en situación de funcionar la iniciativa del señor Antúnez secundada vigorosamente por el ilustrado presidente del Cuerpo médico higienista, Dr. Ronquillo y por el Jefe de la Sección administrativa, vicepresidente del Cuerpo Dr. Castells» 26 .

Por fin, tras largos años de reivindicaciones, se hacía realidad la vieja aspiración de un hospital destinado únicamente a las enfermedades derivadas de la prostitución. Sin embargo, hay que destacar que la creación de este hospital no hubiera sido posible sin la participación entusiasta del entonces gobernador civil Luis Antúnez y Monzón, quien tomó posesión de dicha plaza en Barcelona el 16 de junio de 1886, habiendo sido con anterioridad gobernador civil de las provincias de Orense, Córdoba, Alicante y Lérida. Su mandato significó para Barcelona la realización de mejoras patentes, tanto en el ámbito de las obras públicas, ya que fue promotor de la construcción del Hospital Clínico, de la red de alcantarillado de Gràcia o del saneamiento del delta y llano del Llobregat; como en el ámbito de la higiene pública, en el que tuvo una actuación destacada combatiendo algunas epidemias de fiebre tifoidea declaradas en Sant Adrià del Besós y Manresa y efectuando gran cantidad de donativos a diversas instituciones hospitalarias.

Por lo que respecta a los asuntos relacionados con la prostitución, fue una persona muy sensible con respecto a las situaciones de desamparo, injusticia y pobreza que presidían la vida de la mayoría de mujeres prostitutas. A tal efecto, mandó crear una Junta Benéfica y Administrativa destinada a arbitrar y recaudar fondos destinados a la construcción del nuevo hospital de enfermedades venéreas:

«La erección de un hospital para todos los padecimientos venéreos y sifilíticos se impone ya como de necesidad urgentísima; y la moralidad, la humanidad y la higiene lo reclaman de consuno. La provincia de Barcelona [...] no puede padecer por más tiempo de esta reforma trascendental e importantísima, ni ceder a ninguna otra provincia el glorioso timbre de ser la primera a plantearla en la península española» 27.

Ordenó además, en 1888, un nuevo reglamento de la prostitución, de cuyo articulado destacan, especialmente, dos disposiciones: mediante la primera de ellas, se disponía que las amas de mancebía no podían retener a sus pupilas por

26 «El hospital de higiene», La Vanguardia (Barcelona), 17 de septiembre de 1888.

27 Edicto de constitución de la Junta Benéfica y Administrativa del Hospital para enfermas venéreas y sifilíticas, Barcelona, Gobierno Civil de Barcelona, 3 de julio de 1888. 
razón de las deudas contraídas, suprimiéndose de este modo el estado de esclavitud en el que se hallaban muchas prostitutas; en la segunda, se contemplaba la posibilidad de suprimir el pago por derechos de visita médica, "tan pronto como la Junta consiga reunir las cantidades precisas para el sostenimiento de la Inspección sanitaria» ${ }^{28}$.

La inauguración del modesto nosocomio respondió, en un primer momento, a la urgente necesidad de aumentar, aunque fuera de manera interina, el número de camas disponibles en la ciudad Condal, destinadas al tratamiento y curación de las patologías venéreas y sifilíticas. Sin embargo, la idea que guió desde el principio a sus promotores fue la de construir un hospital donde tratar este tipo de enfermedades, llegándose a elaborar, durante el mandato del gobernador Luis Antúnez, un detallado anteproyecto de construcción por una de las personalidades más decisivas en la mejora de las condiciones de salubridad de Barcelona, el arquitecto e ingeniero de caminos Pedro García Faria ${ }^{29}$.

Desafortunadamente, el tan ansiado hospital nunca llegó a construirse y, lo que es peor, el modesto dispensario de Nuestra Señora de las Mercedes fue clausurado por el Ayuntamiento barcelonés en marzo de 1891. Para ello, no hizo falta esgrimir otras razones que las de carácter administrativo, tales como la supuesta reorganización de los servicios de higiene y salubridad, la próxima (aunque siempre aplazada) resolución del proyecto de construcción del nuevo hospital, o el ahorro que, para la Hacienda municipal, representaba la supresión del servicio. No faltaron tampoco afirmaciones infundadas y tendenciosas en referencia a que el hospital "constituía un foco de infección que puede hasta perjudicar al vecindario y que las infelices albergadas en él en vez de curarse han de resultar perjudicadas ${ }^{30}$. Afirmaciones que, por otra parte, nos recuerdan otras más cercanas en el tiempo, pero igualmente carentes de humanidad, solidaridad y sensibilidad, y que vienen pronunciándose en los barrios de muchas ciudades españolas, a propósito del intento de establecer en esos barrios un centro de día para personas con problemas de drogadicción.

Mientras el pequeño dispensario prestó sus servicios, proporcionó asilo, manutención y vestido a todas aquellas mujeres prostitutas carentes de medios económicos que les permitieran curarse de su enfermedad, con lo cual los estragos producidos por el contagio de enfermedades venéreas quedaron de algún modo reducidos. Por otra parte, el trato personal a las prostitutas distó mucho del que se les venía dispensando en las sombrías salas del hospital de la

28 RONQUillo Y MORER, Carlos: Discurso leído en el acto de inauguración del Dispensario y enfermerías de afecciones venéreas y sifilíticas, Barcelona, Tipografía de J. Balmas Planas, 1888.

29 García Faria, Pedro: «Anteproyecto de un hospital para enfermedades especiales destinado a la provincia de Barcelona. 1888", Revista de Higiene y Policía Sanitaria (Barcelona), 1890, pp. 97-102, 134-139, 167-169, 193-195, 223, 278-281 y 295-296; 1891, pp. 39-40, 71-72, 100-102 y $118-120 ; 1892$, pp. 79-80;124; 163 y 172 .

30 CASTElls BAllespí, Federico: «El hospital de Nuestra Señora de las Mercedes (2a parte)», Revista de Higiene y Policía Sanitaria (Barcelona), 1891, pp. 146-148. 
Santa Cruz donde, además de ser atendidas con repugnancia, eran anatematizadas por el personal religioso, en el probable caso de que se resistieran a los denodados intentos de proselitismo que practicaba asiduamente dicho personal. En este sentido, en el dispensario de Nuestra Señora de las Mercedes y en la Sección de Higiene especial barcelonesa, no parece que se persiguiera tanto la «redención" de la prostituta como la rehabilitación de sus actos vitales, aunque es probable que, en ocasiones, no se pudiera establecer una frontera clara entre los contenidos científicos y los aspectos morales, especialmente, cuando el análisis de la situación social de la prostituta rebasaba los límites de la reglamentación para quedar en manos de la conciencia del médico.

Por otra parte, la existencia del pequeño nosocomio hacía posible el ingreso, por prescripción facultativa del presidente de la Sección de Higiene especial, de la prostituta enferma, cosa que no ocurría en el hospital de la Santa Cruz, ya que sus estatutos impedían el ingreso de un enfermo contra su voluntad. Esta situación fue en muchas ocasiones motivo de controversia, ya que la actuación médica de recluir y aislar a la enferma - aun contra su voluntad - hasta su completa curación en aras de impedir el contagio de la enfermedad, podía representar un delito de detención arbitraria, con lo que no quedaba claro el límite legal de la actuación médica, pese a ser éste el resultado de una acción emanada de la reglamentación vigente.

En definitiva, con el traspaso de los servicios de Higiene al Ayuntamiento y con el cierre del dispensario de Nuestra Señora de las Mercedes, quedaron sin efecto toda una serie de costosas y elaboradas iniciativas destinadas a mejorar la atención médica y social de la prostitución, que se habían venido llevando a cabo desde 1881, volviéndose a una situación que ya se creía superada. A la espera de la construcción de un hospital de enfermedades infecto-contagiosas, anexo al nuevo manicomio que se había de construir en el municipio de Horta (anexionado a Barcelona en 1904), las pútridas salas del hospital de la Santa Cruz volvieron a albergar, de manera precaria y con un número de camas insuficiente, a las meretrices enfermas carentes de medios económicos. Mientras, la interinidad con que se sucedieron algunos cambios políticos en el Ayuntamiento barcelonés determinó que el funcionamiento normal del servicio de Higiene de la prostitución se viera alterado por diversos trastornos organizativos. Este conglomerado de desatinos propició el aumento de curaciones en su domicilio de las prostitutas enfermas, y cierta relajación en el control de las mancebías y de la prostitución clandestina, con el consiguiente incremento del número de contagios de enfermedades venéreas y sifilíticas en la ciudad.

De esta manera, el criterio político - que siempre prevaleció sobre cualquier otra consideración - borró de un plumazo el trabajo que durante muchos años dedicaron los componentes del Cuerpo médico higienista barcelonés al estudio y tratamiento socio-sanitario de la prostitución. Únicamente, el nombramiento de Carlos Ronquillo como Jefe de la Sección cuarta del Cuerpo médico municipal en junio de 1891 , imprimió nuevos bríos al funcionamiento 
de la Sección de Higiene especial dependiente del Consistorio barcelonés, si bien ésta nunca alcanzó los niveles de funcionamiento y atención que demandaba el crecimiento urbano y social de la Barcelona de finales del siglo XIX, como se desprende de la lectura de la Memoria económica de dicha Sección para los años 1891 y 1892, redactada por el propio Ronquillo.

Apenas dos años más tarde, la Real Orden de 6 de diciembre de 1892 volvía a transferir las competencias sobre la higiene de la prostitución a los Gobiernos civiles, con lo cual, una vez más, los designios políticos desmontaban el armazón apenas reconstruido de la Sección de Higiene especial de Barcelona. Este cambio legal determinó, en 1893, el paso del doctor Carlos Ronquillo, como médico numerario, a la Sección de Beneficencia municipal, donde continuó su labor higiénica, esta vez en el campo de la beneficencia domiciliaria. En uno de sus últimos escritos, fruto de las visitas que efectuaba a los hogares más pobres, Ronquillo describió con amargura todas las manifestaciones de pobreza, miseria y enfermedad en que se halla sumida buena parte de la población obrera barcelonesa, incorporando un alegato a la desidia y al abandono en que se encontraban algunas de las medidas sociales y sanitarias destinadas a paliarlas:

«Y todas éstas y otras mil manifestaciones que el médico observa en la Beneficencia domiciliaria, son ecos de la conciencia pública exigiendo la reglamentación de las nodrizas, la higiene de la prostitución, la profilaxis industrial, la higiene escolar, la justicia sanitaria en las viviendas insalubres, las exigencias de la hospitalización, y, como síntesis, abrazando y fundiendo tan variada gama de las ciencias de la salud, la sociología, tan desatendida y no mencionada en ningún reglamento médico municipal31.

Carlos Ronquillo falleció en Barcelona el 17 de febrero de 1900. Con la llegada del nuevo siglo, los avances científicos en materia de microbiología y vacunaciones, las sucesivas legislaciones que, sobre materia sanitaria, se fueron promulgando y la lucha, ardua e infatigable, del movimiento obrero destinada a lograr las necesarias mejoras en sus condiciones de vida y en sus derechos socio-laborales (trabajo, vivienda, enseñanza), marcarían el convulso transcurso histórico de las primeras décadas de la centuria, en la cada vez más industrializada y populosa capital catalana. Respecto al tratamiento socio-sanitario de la prostitución, los avances científicos en el tratamiento de las enfermedades venéreas y sifilíticas no estuvieron acompañados de una actuación social paralela que mitigara la terrible situación del gran número de mujeres que ejercían la prostitución, como única salida económica a la angustiosa precariedad en la que malvivían.

Por último, cabe destacar la labor humanitaria que, a pesar de los continuos cambios políticos y administrativos, desempeñaron los médicos higienis-

31 RONQUILlo MORER, Carlos: «Idiosincrasia barcelonesa», Revista de Higiene y Policía Sanitaria (Barcelona), 1893, pp. 81-83.

Hispania, LXIV/3, núm. 218 (2004) 897-922 
tas, desde las Secciones de Higiene, en el tratamiento social de la prostitución. Gracias a este modo de proceder, se pudieron paliar, de alguna manera, las tremendas carencias sociales implícitas en todas las reglamentaciones que trataron de regular y controlar el ejercicio de la prostitución, sin aportar otros contenidos que los aspectos sancionadores y punitivos como medidas coercitivas al incumplimiento de la normativa.

De todo ello da testimonio la gran labor médica y humana del doctor Carlos Ronquillo y Morer al frente de la Sección de Higiene especial de Barcelona, pionera, en España, en la incorporación al tratamiento higiénico de la prostitución del estudio sociológico, de sus causas y de los remedios sociales necesarios para evitarla, no tan sólo como elemento de transmisión de patologías venéreas, sino también como una forma de rescatar la dignidad personal de la mujer prostituta. 